\title{
Remote Patient Management in Peritoneal Dialysis: Impact on Clinician's Practice and Behavior
}

\author{
Carlo Crepaldia • Anna Giuliania • Sabrina Milan Manania • \\ Nicola Marchionna ${ }^{a} \cdot$ Paola Piasentin ${ }^{a} \cdot$ Claudio Ronco $^{a-c}$ \\ ${ }^{\text {a } D e p a r t m e n t ~ o f ~ N e p h r o l o g y, ~ D i a l y s i s ~ a n d ~ T r a n s p l a n t, ~ S a n ~ B o r t o l o ~ H o s p i t a l, ~ V i c e n z a, ~ I t a l y ; ~}$ \\ ${ }^{b}$ International Renal Research Institute Vicenza (IRRIV), Vicenza, Italy; ${ }^{C}$ University of Padova, \\ Padova, Italy
}

\begin{abstract}
Peritoneal dialysis (PD) is a self-administered chronic renal replacement therapy. It is a homebased therapy, and thus subject to the risk of discrepancy between prescribed dose and effective dialysis delivery. Till now automated peritoneal dialysis (APD) cyclers have recorded the dialysis treatments on a card that patients bring to the hospital for consultation in the PD unit. This card contains the operative parameters of each APD session. Recently, Baxter Healthcare developed a cloud-based tool for remote patient and treatment management. The new platform named Sharesource ${ }^{\circledR}$ embedded into the cycler HOMECHOICE CLARIA ${ }^{\circledR}$ allows to overcome the problems related to poor compliance and feeling of uncertainty by the patient, reducing the number of hospital visits and the workload for physician and nurses of the PD Unit. This new system uploads all treatment information to a secure cloud-based software. The 2-way communication platform gives remote visibility to patient's treatment and allows for feedback and correction of inadequate treatment program. Remote patient management (RPM) allows to visualize the course of home PD day after day, evaluating adherence to prescription, possible alarms during treatment, drainage times, and ultrafiltration amount. The evaluation of all the data can be done by the physician at his desk in the Hospital in front of the computer. RPM allows a patient's dialytic management in real time and enables the nephrologist to remotely modify treatment operative parameters, leaving the patient at home saving kilometers, money and time. In this chapter, we describe a simple algorithm used in our unit to define alarm thresholds and to describe actions to be instituted to correct any possible problem occurring during APD.




\section{Introduction}

The rising prevalence of chronic diseases, such as hypertension, heart failure, diabetes, end-stage kidney disease, is a global concern. Generally, incurable and ongoing, chronic diseases affect approximately 133 million Americans, representing more than $40 \%$ of the total population of this country. These conditions require intensive and ongoing medical assistance to maximize outcomes and avoid the risk of frequent flare-ups and hospitalizations, which increase the cost of healthcare [1].

The hospitalization rates for chronic diseases can be considered a negative marker of the quality of outpatient and community-based care; however, appropriate care can diminish costs and improve outcomes, thereby reducing hospitalizations.

Several interventions such as discharge planning, in-home care, continuity of care, advanced access scheduling, self-management support interventions, specialized nursing practice, electronic tools for health information exchange, and health technologies have been suggested in the chronic disease. In particular, health technologies should be considered an important part of chronic disease management in the community.

\section{Telemedicine and Remote Monitoring}

Telemedicine (TM) is an innovative tool that provides remote transmission, interpretation, and storage of clinical parameters and useful diagnostic images. A subset of TM is remote monitoring (RM) where physiological variables or images can be sent to a central monitoring center for review and intervention of the care team. It allows for accurate home monitoring of patients, enabling the team to improve care through prevention and early identification of problems, with consequent timely interventions. In this view, TM and RM may have important reflections on prognosis, outcomes, and quality of life. RM has been proved to be useful in chronic diseases where frequent controls are particularly useful such as in heart failure, diabetes, and hypertension. The possibility of keeping patients out of hospital, supporting their home care by RM may contribute not only in preventing complications, but also in reducing direct and indirect costs.

TM allows healthcare providers to remotely diagnose and treat patients using telecommunications as an alternative to in-person visits, both reducing costs and in-facility congestion. While today's TM services are limited in scope, remote patient management (RPM) technology could empower actionable feed- 
back, improve the quality of care, and increase remote patient healthcare access [2]. A 2015 retrospective evaluation of a RM program with 348 heart failure patients was associated with significantly lower hospitalization rates of up to 90 days and significantly lower mortality rates over 120 days of the program [3]. In 2017, Abbott Laboratories Inc. released data from a study showing that patients who scan more frequently using the company's remote glucose monitoring system, FreeStyle Libre, have improved average glucose levels. In cardiology, RM or TM systems have potential advantages such as early detection of cardiovascular events and early response to technical problems of the device or changes in the patient's clinical status. TM may be a potential alternative to reduce the number of in-office visits to hospital, thereby optimizing medical resources. In the last decade, several studies have shown that TM is as effective as traditional follow-up in hospital [4]. Very interesting is a meta-analysis of intervention studies designed to identify the effects of remote home management on patients with chronic kidney disease (CKD): the available data indicate that remote home management may be a novel and effective disease management strategy for improving CKD patients' quality of life and influencing their attitudes and behaviors [5]. Only in the last 20 years, the concept of TM and RM have begun to interest the nephrologists and the field of dialysis. Nakamoto [6] has published a paper about peritoneal dialysis (PD) and RM. In 1999, he reported on a TM system (version 1.0) that used an automated peritoneal dialysis (APD) machine to collect data on patients with end-stage renal disease. After 2002, he focused on using cellular telephones in a new TM system (version 2.0) to monitor patient data at home, including blood pressure, heart rate, body weight, urine volume, and blood glucose. By 2003, he had developed a fully automatic system called Iconverter (version 3.0) to collect data from a fully automatic device and send it via cellular telephone [6]. Gallar [7] published a paper with his evaluation about the use of TM in the long-term control of stable patients undergoing PD at home. From September 2003 to August 2005, patients were randomly selected from current cases and invited to join the study group A, in which they had TM support. Unselected patients, or those who refused the invitation, were placed in study group B, and used for comparison. There were 25 patients in group A and 32 patients in group B. Videoconferencing equipment was installed in each patient's home, connected to a videoconferencing unit at the hospital by 3 ISDN lines. Patients in group A were followed for a mean of 8 months (range 3-24) with alternate months of teleconsultations and hospital visits. A total of 172 teleconsultations were conducted. A mean of 22 min (SD 9) were spent on each teleconsultation, significantly less than in hospital consultations, which took a mean of $33 \mathrm{~min}$ (SD 8; $p<0.01$ ). In 148 teleconsultations $(89 \%)$, medical treatment was modified. In 4 cases (2\%), patients needed a hospital visit. In all in- 
stances (100\%), the condition of the catheter exit site and the presence of edema could be evaluated. In group A, the estimated cost of TM was euro198 and that of a hospital visit was euro 177. The mean hospitalization rate was 2.2 days/patient/year in group A and 5.7 days/patient/year in group B $(p<0.05)$. Home TM appears to be clinically useful in the long-term follow-up of stable patients undergoing PD, and the costs and savings also seem to be encouraging [7]. In 2012, Nayak et al. [8] published their experience about the use of a PD RM system in India. This study showed that a mobile-driven RM ensured in a rural population $\mathrm{PD}$ technique failures, peritonitis rates, exit-site infections comparable with an urban population living close to the Nephrology hub hospital. Patient survival at 5 years was even significantly better in rural patients [8].

\section{SHARESOURCE ${ }^{\circledR}$ Platform}

HOMECHOICE CLARIA ${ }^{\circledR}$ with Sharesource ${ }^{\circledR}$ is a Baxter APD system. HOMECHOICE CLARIA ${ }^{\circledR}$ is an APD cycler which performs dialysis overnight while the patient sleeps. The technology under review is the addition of Sharesource $^{\circledR}$ to the existing HOMECHOICE ${ }^{\circledR}$ device. The combined unit uploads all treatment information to a secure cloud-based platform (Sharesource ${ }^{\circledR}$ ) where healthcare professionals (HCPs) can access it and monitor patient treatment. It is a 2-way platform that gives HCPs remote visibility of a patient's treatment outcomes such as fill volume, dwell time, and total therapy time. Any issues are highlighted using a flag system, and these flags can be customized according to the individual renal unit's protocols. The system allows the HCPs to change the devices' default settings such as alarms and prescription parameters. The data from Sharesource ${ }^{\circledR}$ is transmitted from a modem within the APD cycler. Only a weak mobile telephone signal is required. At clinics, access to the SHARESOURCE ${ }^{\circledR}$ portal requires a computer and the internet. Baxter launched their new APD system in 2015 - HOMECHOICE CLARIA ${ }^{\circledR}$ with Sharesource ${ }^{\circledR}$. This technology enables RPM of patients having APD at home. HOMECHOICE CLARIA $^{\circledR}$ with Sharesource ${ }^{\circledR}$ is a class 2 b device. HOMECHOICE CLARIA ${ }^{\circledR}$ with Sharesource ${ }^{\circledR}$ is intended for use in people with end-stage renal failure. End-stage renal failure is when $\mathrm{CKD}$ has progressed so far that renal replacement therapy (regular dialysis treatment or kidney transplantation) is required to maintain life. Kidney transplantation is not suitable for all people receiving dialysis. Under review is the addition of Sharesource ${ }^{\circledR}$ to the existing HOMECHOICE ${ }^{\circledR}$ device. Sharesource ${ }^{\circledR}$ is embedded within the HOMECHOICE CLARIA $^{\circledR}$ cycler. The Sharesource ${ }^{\circledR}$ dashboard shows clinicians which patients have received dialysis, whether there were any issues and the nature of the issues. 
It also shows how patients' prescriptions compare to their delivered dialysis dose. The 2-way connectivity allows clinicians to change the devices' default settings such as alarms, prescription parameters such as fill volume, dwell time, and total therapy time. Problems are highlighted using a flag system, and these flags can be customized according to the individual unit's protocols. Only authorized users are able to remotely view information from the SHARESOURCE ${ }^{\circledR}$ platform and manage treatment. All hospitals using the technology have a signed data-sharing agreement with Baxter. The patient also has to consent to the use of the Sharesource ${ }^{\circledR}$ feature of the machine. If patients do not give this consent, the APD cycler can still be used, but their treatment data will not be uploaded to the SHARESOURCE ${ }^{\circledR}$ platform. The manufacturer states that Sharesource ${ }^{\circledR}$ is the only 2-way remote management system available for dialysis patients who treat themselves at home. A high level search conducted by Healthcare Improvement Scotland did not highlight any other similar technologies. Some studies on the use of TM in patients with kidney disease were identified, but these generally involved phones (for calls or texts), videoconferencing, or applications/software on tablet computers.

\section{RM in APD: Adherence to Dialysis Prescription}

With increasing numbers of ESRD patients in need of renal replacement therapy and the need to expand dialysis delivery in home settings away from overburdened hospital and tertiary care settings, there is renewed interest in outcomes in patients who are established on PD regimes. Adherence to treatment is of paramount importance as non-adherence has been shown to have major consequences including an increased risk of mortality and hospitalization in patients on $\mathrm{HD}$ and in $\mathrm{PD}$ which in turn lead to increased costs and expenditure for patient care. Researchers often use the terms compliance and adherence interchangeably, although they have slightly different implications. Compliance, principally used extensively in older literature, has drawn criticism for its emphasis on medical authority and an implication for patients as passive recipients of care. In response, the term adherence was introduced to recognize patients' right to choose whether or not to follow advice, calling attention to the importance of patients' active participation in their treatment regimes [9]. PD patients are required to adhere to a demanding dialysis regime that involves regular manual exchanges at least thrice daily (in the case of continuous ambulatory peritoneal dialysis, CAPD) or long overnight dialysis exchange (in the case of APD), as well as lifelong changes in lifestyle related to diet, intake of multiple medications, and safety and preventive measures. Overall, non-adherence rates ranged 
from 2.6 to $85.2 \%$ [9]. In general, although non-adherence rates were somewhat higher for medication and diet compared to dialysis procedures, the rates of missing dialysis exchanges/sessions were far from negligible. In most studies, more than $20 \%$ of PD patients performed less than $90 \%$ of prescribed exchanges. Given the potential repercussions of non-adherence such as technique failure, peritonitis, and hospitalization, these rates are alarming [10, 11]. A system to detect non-adherent patient to PD dialysis treatment has been found with the use of a card recording the APD treatments of the patient. Currently, no reliable system has been discovered to measure the rate of adherence of CAPD patients. During the scheduled visits, nurses and/or nephrologists upload the records of the APD treatment from the card and evaluate parameters like adherence, ultrafiltration (UF) of APD treatments, number and type of alarms during the treatment. This procedure takes long time to evaluate all these parameters. The new SHARESOURCE ${ }^{\circledR}$ platform from the first screen allows to detect from the dashboard problems as non-adherence to the prescription with APD treatments not carried out, treatments characterized by numerous alarms or by a UF lower than expected. The patient is aware and informed that the doctor and/or nurses can see their treatments day after day from the center and is therefore encouraged to perform them regularly. Our experience with SHARESOURCE ${ }^{\circledR}$ platform has found that less than $1 \%$ of APD treatments are not carried out in the absence of technical problems. These data are much better than those reported in the literature and demonstrate the effectiveness of the platform as a deterrent to non-adherence with the APD prescription.

\section{RM in APD: APD UF}

Fluid overload is very common among PD patients $[12,13]$. A daily monitoring of dialytic UF with RM is very helpful in the management of a PD population.

Optimization of dwell time (according to the result of PET test), change of dialytic solution osmolarity, and variation of treatment time may reduce patient's fluid overload. In patients with reduced residual renal function, the introduction of a CCPD1 using an icodextrin or hypertonic glucose exchange during the daytime may be helpful to normalize patient hydration status. The patient may give information of his/her body weight, registering it on their display screen. This data will be seen the day after by the PD unit nurses or the nephrologists on the SHARESOURCE ${ }^{\circledR}$ platform.

A daily evaluation of patient UF and body weight is helpful. If UF and patient's body weight remain stable we can suppose the fluid intake and diuresis are also stable. 


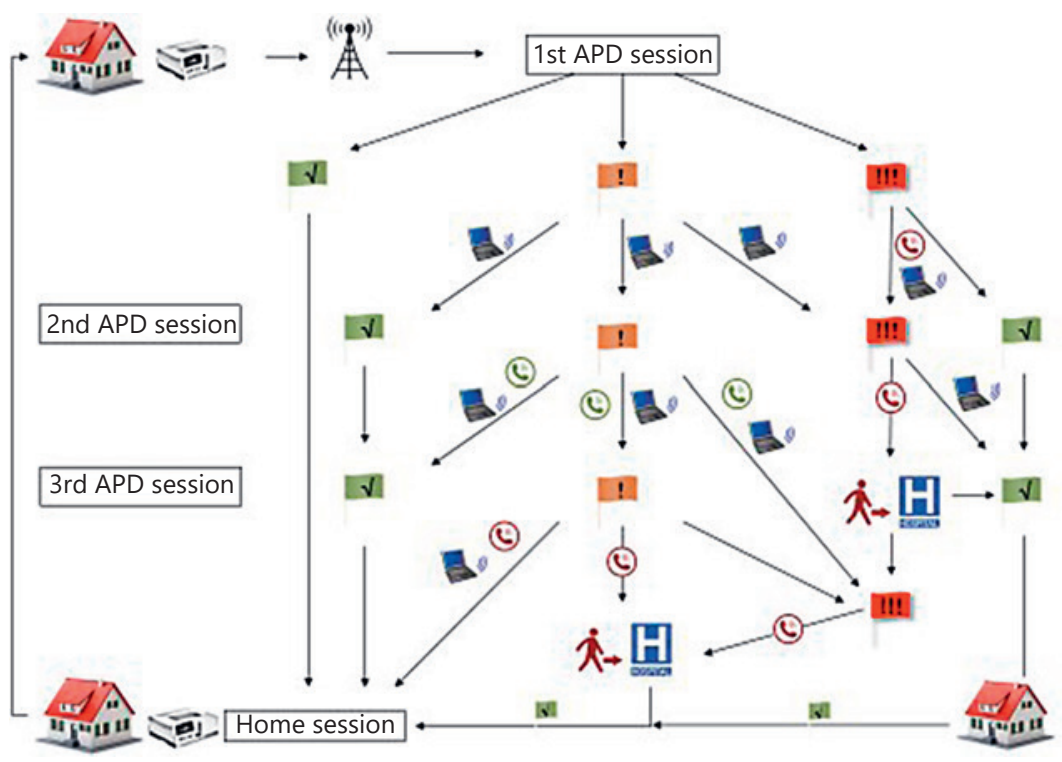

Fig. 1. Algorithm for RM-APD patients to describe different types of alarms and consequent actions prompted by the alarm codes (reprinted with permission from [15]). RM, remote monitoring; APD, ambulatory peritoneal disease. This algorithm is based on the different flags on the SHARESOURCE ${ }^{\oplus}$ platform (Fig. 2).

\section{RM in APD: Clinical Management of Alarms}

Sleep pattern disturbance is a problem among dialysis patients. Of the factors reported to interfere with sleep, treatment-related factors such as alarms and other machine-related problems were, by far, the most predominant [14]. Daily observation of alarms during APD treatment allows the nephrologist to modify the operative parameters of the dialysis treatment. Changes to the dialysate fill of abdomen or the requested UF, possible adoption of a Tidal dialysis, modification of the percentage of Tidal, are different tools to change the APD prescription remotely. An evaluation about number and quality of alarms may induce the nephrologist to confirm or to change again the APD program. SHARESOURCE ${ }^{\circledR}$ platform helps the nephrologist and the patient: the nephrologist in $24 \mathrm{~h}$ can modify the APD program from his desk in the Hospital, and the patient avoid trip from home to the dialysis unit. This aspect is important in Europe, becomes of fundamental importance in countries with large territorial exten- 


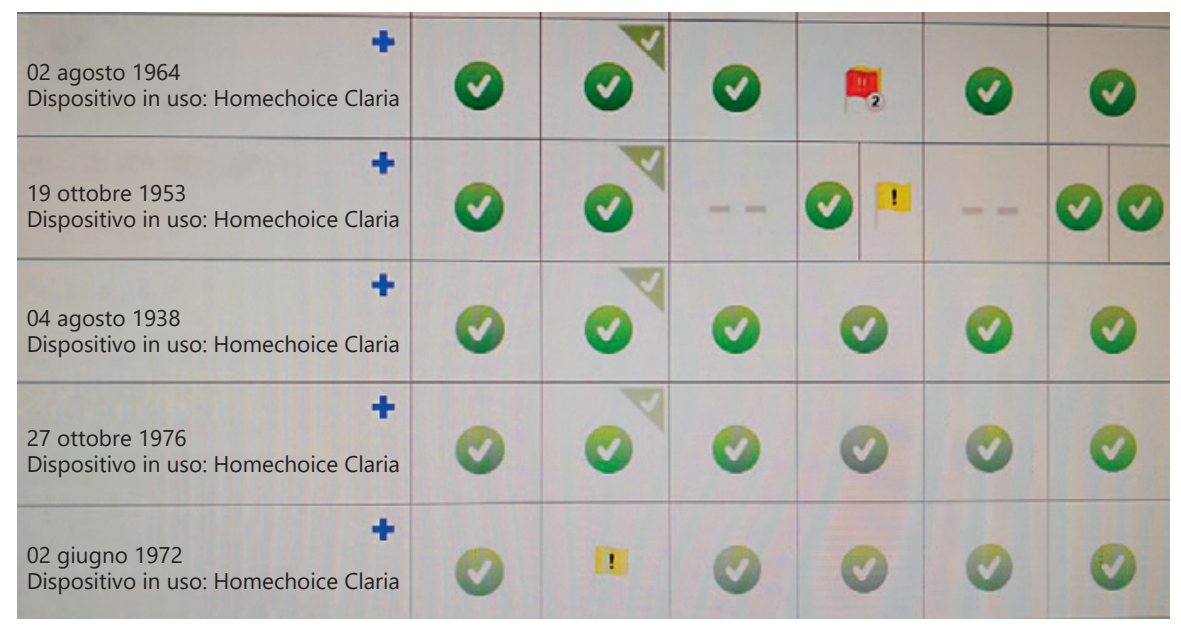

Fig. 2. Sharesource ${ }^{\circledR}$ dashboard with flags alert.

sions (i.e., USA, Canada, Australia, New Zealand, Mexico) where the distance between patient's home and dialysis center is noticeable.

The treatment is device-transmitted to the PD center, when the patient switches off the cycler and the care team in the morning is able to view the night treatments of all patients in the platform. The sessions without significant problems appear with a color-code green, however the treatments with problems identified by our settings are represented with yellow or red flags. Every PD Unit can manage the Flag Alerts by choosing among those proposed by SHARESOURCE ${ }^{\circledR}$ platform and among the possible issue flags chosen if the issue is important (yellow flag) or very relevant (red flag). Our specific interventions based on the platform observation follow the algorithm reported in Figure 1.

The daily observation of Sharesource ${ }^{\circledR}$ dashboard by nurses and physician of the PD unit is obviously addressed to the yellow and red flags. The flags alert indicates a decision path (Fig. 2). In our unit, the procedures adopted according to the type of alarms are described in Table 1.

In our PD unit, we decided the following management of flags:

\section{Treatment Time}

Notice appears if the actual duration of night treatment is $30 \mathrm{~min}$ shorter than the set threshold value (yellow flag).

\section{Treatment Modifications}

a. Volume of therapy lost: warning appears if the actual therapy volume is $10 \%$ lower than the threshold value of the total therapy volume set (red flag); 
Table 1. Flags alert and decision path

\begin{tabular}{ll}
\hline Flag & First action by nurses \\
\hline $\begin{array}{l}\text { Treatment time lost }>30 \mathrm{~min} \\
\text { Delivery volume lost }>10 \%\end{array}$ & Call the patient, verify compliance \\
Bypass drain $>2$ times & Call the patient, verify compliance \\
Bypass dwell/fill $>2$ & Call the patient, exclude overfilling \\
No. of events $>5$ & Call the patient, verify compliance \\
No. of events $>10$ & Suggest avoid constipation/use heparin \\
\hline Flag & Suggest avoid constipation/use heparin \\
\hline Treatment time lost $>30$ min & Second action by physician \\
Delivery volume lost $>10 \%$ & Remotely change APD - in person visit \\
Bypass drain $>2$ times & Remotely change APD - in person visit \\
Bypass dwell/fill $>2$ & Remotely change APD - in person visit \\
No. of events $>5$ & Remotely change APD - in person visit \\
No. of events $>10$ & Remote reduction of tidal\% or UF - in person visit \\
\hline
\end{tabular}

b. Early discharge term: a warning appears when a drain is bypassed. The warning will be displayed when the number of bypassed drains is equal to or greater than our threshold value (red flag).

\section{Patient Intervention}

Bypass number of the load/stop phase: a warning appears if the number of times the patient has performed a bypass during the stop or the load is equal to or greater than 2 our threshold value (red flag).

\section{System Errors}

Events during treatment: the notice appears if the number of events that occurred during treatment is equal to or greater than 5 (yellow flag) or 10 (red flag), the set threshold value.

Line alarms are usually related to the kinking of catheter or obstruction by fibrin. In some cases, if the catheter performance is considered to be low, tidal percentage can be modified directly by remote, so that lower volume drainage is required during the night. Alarms, such as UF insufficient, negative UF or insufficient drain volume, are set off when drainage is over and drained volume is inferior. In this case, the same suggestion of line alarms are given by the nurse and if alarms are persistent, the patient needs to come to the center to exclude volume overload and perform a manual drainage. If it happens during the first home treatments, when the standard volume of ultrafiltrate is still to be established, it can be directly changed by remote. 


\section{Disclosure Statement}

No authors have reported a conflict of interest. This manuscript was seen and approved by all authors listed. It is not under consideration for publication elsewhere in a similar form, in any language, except in abstract form.

\section{References}

1 Raghupathi W, Raghupathi V: An empirical study of chronic diseases in the United States: a visual analytics approach. Int J Environ Res Public Health 2018;15:431.

2 Remote Patient Monitoring: A Telemedicine Solution to Chronic Disease September 13, 2017 Biotricity.

3 Agboola S, Jethwani K, Khateeb K, Moore S, Kvedar J: Heart failure remote monitoring: evidence from the retrospective evaluation of a real-world remote monitoring program. J Med Internet Res 2015;17:e101.

4 Cronin EM, Varma N: Remote monitoring of cardiovascular implanted electronic devices: a paradigm shift for the 21st century. Expert Rev Med Devices 2012;9:367-376.

5 He T, Liu X, Li Y, Wu Q, Liu M, Yuan H: Remote home management for chronic kidney disease: a systematic review. J Telemed Telecare 2017;23: $3-13$.

6 Nakamoto H: Telemedicine system for patients on continuous ambulatory peritoneal dialysis. Perit Dial Int 2007; 27(suppl 2):S21-S26.

7 Gallar P, Vigil A, Rodriguez I, Ortega O, Gutierrez M, Hurtado J, Oliet A, Ortiz M, Mon C, Herrero JC, Lentisco C: Two-year experience with telemedicine in the follow-up of patients in home peritoneal dialysis. J Telemed Telecare 2007;13: 288-292.
8 Nayak A, Karopadi A, Antony S, Sreepada S, Nayak KS: Use of a peritoneal dialysis remote monitoring system in India. Perit Dial Int 2012;32: 2200-2014.

9 Griva K, Lai AY, Lim HA, Yu Z, Foo MW, Newman SP: Non-adherence in patients on peritoneal dialysis: a systematic review. PLoS One 2014; 9:e89001.

10 Bernardini J, Piraino B: Compliance in CAPD and CCPD patients as measured by supply inventories during home visits. Am J Kidney Dis 1998;31: 101-107.

11 Bernardini J, Nagy M, Piraino B: Pattern of noncompliance with dialysis exchanges in peritoneal dialysis patients. Am J Kidney Dis 2000;35:11041110.

12 Huyge L, Locking-Cusolito H: Incidence of sleep pattern disturbance in a peritoneal dialysis sample. Adv Perit Dial 2000;16:156-162.

13 Shu Y, Liu J, Zeng X, Hong HG, Li Y, Zhong H, Ma L, Fu P: The effect of overhydration on mortality and technique failure among peritoneal dialysis patients: a systematic review and metaanalysis. Blood Purif 2018;46:350-358.

14 Van Biesen W, Williams JD, Covic AC, Fan S, Claes K, Lichodziejewska-Niemierko M, Verger C, Steiger J, Schoder V, Wabel P, Gauly A, Himmele R; EuroBCM Study Group: Fluid status in peritoneal dialysis patients: the European body composition monitoring (EuroBCM) study cohort. PLoS One 2011;6: e17148.

15 Milan Manani S, Rosner MH, Virzì GM, Giuliani A, Berti, Crepaldi C, Ronco C: Longitudinal experience with remote monitoring for automated peritoneal dialysis patients. Nephron 2019;1-9.

Carlo Crepaldi

Department of Nephrology, Dialysis and Transplant

San Bortolo Hospital

IT-36100 Vicenza (Italy)

E-Mail carlo.crepaldi@aulss8.veneto.it 